\title{
Artemether Sublingual Spray
}

National Cancer Institute

\section{Source}

National Cancer Institute. Artemether Sublingual Spray. NCI Thesaurus. Code C118504.

A sublingual spray containing artemether, a semisynthetic derivative of artemisinin, an endoperoxide extracted from the Chinese herb qinghaosu (Artemisia annua or annual wormwood), with antiparasitic and potential antineoplastic activity. Upon sublingual application of the spray, artemether exerts its antineoplastic activity through as of yet not fully elucidated mechanism(s) of action. This agent binds to heme molecules inside cells, thereby inducing reactive oxygen species (ROS)-mediated damage which selectively kills cancer cells. In addition, artemether appears to target and modulate the expression of various proteins involved in cancer cell proliferation, angiogenesis, invasion and metastasis. Also, this agent depletes T regulatory cells, and modulates the production of inflammatory cytokines, such as interleukin-4 and interferon-gamma. Altogether, this inhibits tumor cell proliferation. The sublingual spray allows faster absorption of a higher percentage of the artemether dose, when compared to the oral form, as it avoids first pass metabolism; this results in an increased efficacy. 